\title{
Is It Possible to Delay or Prevent Age-Related Cognitive Decline?
}

\author{
Jean-Pierre Michel* \\ Academic Geriatric Department, Geneva University, Geneva, Switzerland
}

\begin{abstract}
Already in the 90s, Khachaturian stated that postponing dementia onset by five years would decrease the prevalence of the late onset dementia by $50 \%$. After two decades of lack of success in dementia drug discovery and development, and knowing that worldwide, currently 36 million patients have been diagnosed with Alzheimer's disease, a number that will double by 2030 and triple by 2050, the World Health Organization and the Alzheimer's Disease International declared that prevention of cognitive decline was a 'public health priority.' Numerous longitudinal studies and meta-analyses were conducted to analyze the risk and protective factors for dementia. Among the 93 identified risk factors, seven major modifiable ones should be considered: low education, sedentary lifestyle, midlife obesity, midlife smoking, hypertension, diabetes, and midlife depression. Three other important modifiable risk factors should also be added to this list: midlife hypercholesterolemia, late life atrial fibrillation, and chronic kidney disease. After their identification, numerous authors attempted to establish dementia risk scores; however, the proposed values were not convincing. Identifying the possible interventions, able to either postpone or delay dementia has been an important challenge. Observational studies focused on a single life-style intervention increased the global optimism concerning these possibilities. However, a recent extensive literature review of the randomized control trials (RCTs) conducted before 2014 yielded negative results. The first results of RCTs of multimodal interventions (Finnish Geriatric Intervention Study to Prevent Cognitive Impairment and Disability, Multidomain Alzheimer Prevention Study, and Prediva) brought more optimism. Lastly, interventions targeting compounds of beta amyloid started in 2012 and no results have yet been published.
\end{abstract}

Keywords: Cognitive Decline; Dementia; Alzheimer Disease; Brain Reserve; Physical Activity; Mediterranean Diet 


\section{INTRODUCTION}

As early as the 90s, Khachaturian stated that delaying the onset of dementia by five years would decrease the prevalence of late-onset dementia by $50 \% .{ }^{1)}$ After two decades without any major advances in the discovery or development of dementia drugs, ${ }^{2)}$ in a landmark report, the World Health Organization and Alzheimer's Disease International stated that prevention of cognitive decline had become a 'public health priority,' given that, at the time of the publication, 36 million patients worldwide were diagnosed with Alzheimer's disease (AD), and this number was set to double by 2030 and triple by $2050 .{ }^{3)}$ However, surprisingly, the 1975-2015 report of the Framingham Heart study showed a $20 \%$ decrease in dementia incidence for each decade, while the prevalence of overweight and cardiovascular events did not decrease at the same rate, over the same period of time. ${ }^{4)}$ The debate is on-going, since no such decline was observed either in the Rotterdam study or in the low- and middle-resource countries, whose ageing population will grow faster than that of the developed, high-resource countries. ${ }^{5)}$ In this context of 'cautious optimism, we should recall that recent meta-analyses of longitudinal studies have identified numerous risk and protective factors for dementia, ${ }^{6)}$ of which at least seven are modifiable, ${ }^{7)}$ namely: low educational attainment (relative risk [RR], 1.59; 95\% confidence interval [CI], 1.35 to 1.86$)$, sedentary lifestyle $(<30$ minutes of activity, five times per week; RR, 1.82; 95\% CI, 1.19 to 2.78 ), midlife obesity (body mass index [BMI] $>30 \mathrm{~kg} / \mathrm{m}^{2}$; RR, 1.60; 95\% CI, 1.34 to 1.92 ), smoking at midlife (RR, $1.59 ; 95 \% \mathrm{CI}, 1.15$ to 2.20 ), hypertension ( 35 to 64 years old; RR, 1.61; 95\% CI, 1.16 to 2.24 ), diabetes (20 to 79 years old; RR, 1.46; 95\% CI, 1.20 to 1.77 ), and midlife depression (RR, 1.37; 95\% CI, 1.05 to 1.78). ${ }^{7)}$ Three other important modifiable risk factors deserve to be added to this shortlist ${ }^{8}{ }^{8}$ i.e., serum cholesterol concentration ( $\geq 6.5 \mathrm{mmol} / \mathrm{L}$; odds ratio [OR], 2.1; 95\% CI, 1.0 to 4.4 ), ${ }^{9)}$ late life atrial fibrillation (hazard ratio, 2.61; 95\% CI, 1.05 to 6.47$),{ }^{10)}$ and chronic kidney disease (OR, 1.65; 95\% CI, 1.32 to 2.05$).{ }^{11)}$

After identifying the major modifiable risk factors for dementia, numerous authors attempted to establish dementia risk scores. The first such score was based on the 20-year follow-up of the Cardiovascular Risk Factors, Aging, and Incidence of Dementia (CAIDE) cohort, including 1,409 participants, whose mean age at the start of the study was 50.4 years of age. ${ }^{12)}$ The score included advanced age, low education, female gender, high BMI, high systolic blood pressure, high total cholesterol, and low physical activity. For the participants with the lowest dementia risk score, the estimated risk of late-life dementia was $1 \%$, while the risk reached $16.4 \%$ for those with the highest risk score. ${ }^{12)}$ Application of this score to the Kaiser Permanente cohort, which included 9,480 participants with a baseline age between 40 and 55 years, showed that after 40 years of follow-up, the score allowed stratification of participants into those with 40-year low (9\%) and high (29\%) dementia risk. ${ }^{13)}$ The addition of five other risk factors to the CAIDE score (head trauma, central obesity, depression, poor pulmonary function, and diabetes) did not yield any additional predictive value. ${ }^{13)}$ Another attempt was performed in a UK cohort, comparing the Framingham cardiovascular and stroke scores to the CAIDE dementia risk score after 10 years of follow-up. It appeared that the Framingham cardiovascular and stroke scores better predicted the rapid decline in global cognitive score, semantic fluency, and all tests except memory, as compared to the CAIDE score, which better predicted the rapid decline in reasoning and vocabulary. ${ }^{14)}$

This inventory of the major modifiable dementia risk factors, as well as the composition and results obtained by the proposed dementia risk prediction score, highlight the importance of midlife cardio-, neuro-, and reno-vascular risk factors. As a reminder, these are: midlife obesity, sedentary lifestyle, diabetes, hypercholesterolemia, smoking, hypertension, atrial fibrillation, and chronic kidney disease.

Following on from this list, the main question is then to identify possible interventions to, either postpone and shorten the episode; delay the onset, while living to a more advanced age; or, in the best case scenario, prevent dementia altogether. ${ }^{15)}$ Recently, a complete review and analysis of all trials published before 2013 that were performed in unselected participants at risk of dementia or cognitively impaired, was published. The conclusions of this study showed little evidence of improvement, irrespective of the type of intervention (advice, physical exercise, nutritional supplements, cognitive activity, or training and medications). ${ }^{16)}$

However, this does not justify not searching for a viable alternative. Given the considerable decline in incidence reported in the recent paper based on the 20 year follow-up of the Framingham study, ${ }^{4)}$ it seems necessary to take another look at the new developments in the field of prevention of cognitive decline. Four types of studies need to be analyzed.

\section{OBSERVATIONAL STUDIES FOCUSED ON A SINGLE SPECIFIC LIFESTYLE INTERVENTION}

The first observational study showing the possible impact of fish intake on dementia prevention was published in 1997. The 2.1-year followup of 5,386 participants without dementia, aged over 55 years at baseline, showed that eating fish, an important source of $n-3$ polyunsaturated fatty acids, was inversely related to the incidence of dementia (RR, 0.4; 95\% CI, 0.2 to 0.91 ). ${ }^{17)}$ More recently, a significant positive relationship was observed between fish consumption and functional cortical activity in a population of 142 Japanese workers, suggesting that fish consumption modulates functional activity in the left dorsolateral prefrontal cortex. ${ }^{18)}$ Regarding physical activity, a meta-analysis of 15 prospective studies (mean length of follow-up 5 years) that altogether included 33,816 adults, aged over 65 years (12 cohorts from Canada, China, Europe, Singapore, and the USA), who did not show cognitive impairment at study inclusion, showed that moderate to high physical activity protects significantly against cognitive decline $(\mathrm{P}<0.00001) .{ }^{19)}$ 


\section{RANDOMIZED CONTROL TRIALS OF ONE SPECIFIC NTERVENTION}

The aforementioned survey of randomized control trials (RCTs) published before 2013 yielded negative findings. These negative results were explained by numerous interfering factors, such as the very heterogeneous study designs, timing of the intervention with regard to the disease onset, small heterogeneous samples, various targeted groups, and variable durations of follow-up. ${ }^{16)}$ However, a sub-analysis of the Prevención con Dieta Mediterránea (PREDIMED) study, namely the PREDIMED-Navarra randomized trial, including 522 participants at high vascular risk ( $44.6 \%$ men; age, $74.6 \pm 5.7$ years), compared two types of Mediterranean diet: supplemented with extra-virgin olive oil or supplemented with nuts, versus a low-fat control diet. After 6.5 years of follow-up and adjustment for 15 variables, it appeared that the two supplemented Mediterranean diets had a significant protective effect (from $\mathrm{P}=0.048$ for nuts to $\mathrm{P}=0.0001$ for extra-virgin olive oil supplements) against cognitive decline as assessed by two global cognitive tests (Folstein's Mini Mental State Examination and the Clock drawing test). ${ }^{20)}$

\section{RESULTS OF RANDOMIZED CONTROL TRIALS OF MULTIMODAL INTERVENTIONS}

Currently, there are three ongoing RCTs for the prevention of cognitive impairment based on multidomain interventions, the Finnish Geriatric Intervention Study to Prevent Cognitive Impairment and Disability (FINGER), Multidomain Alzheimer Prevention Study (MAPT), and PreDIVA trials. ${ }^{21)}$ So far, only the results of the FINGER (FINnishGERiatric) study are known. This was a double blind RCT of 1,260 individuals aged 60-77 years with a CAIDE dementia score $>6$. The intervention group $(\mathrm{n}=631)$, which benefited from a multidomain intervention (comprising diet, exercise, cognitive training, vascular risk monitoring) for 2 years was compared to a control group $(n=629)$, who received only general health advice. The primary outcome was the comprehensive neuropsychological test battery (NTB) Z-score (a composite score based on results from 14 tests, calculated as Z-scores, standardized to the baseline mean and standard deviations, with higher scores suggesting better performance). The estimated mean change in the NTB $\mathrm{Z}$-score at 2 years was better $(\mathrm{P}=0.030)$ in the intervention group $(0.20 \pm 0.51)$ compared to the control group $(0.16 \pm 0.51)$. Thus, the FINGER study has shown that a multi-modal intervention could improve or maintain cognitive functioning in at-risk older adults. ${ }^{22)}$ Undoubtedly, the results of the MAPT and PreDIVA will be the focus of much attention after such encouraging results from the FINGER study.

\section{ONGOING INTERVENTIONS TARGETING COMPOUNDS OF BETA AMYLOID}

Up to now, only study protocols have been published for studies in this domain, of which several have just started (between 2012 and 2014).
The studies target autosomal dominant early-onset Alzheimer's disease (two) and asymptomatic Alzheimer's disease (one) ${ }^{21)}$ Results will be eagerly awaited.

\section{CONCLUSION}

How can we conclude this editorial, other than by stressing that regardless of the clinical presentation of the first symptoms of dementia, the major modifiable risk factors remain of cardio-, neuro-, and renovascular origin. Consequently, as a primary priority, health promotion and midlife prevention need to target these. A systematic search of PubMed and the Cochrane Database from inception to July 2014 identified 19,906 published reviews on the same topic, as this editorial. Based on 323 selected papers, the authors of the review established two kinds of grade 1 recommendations. Dietary recommendations including intake of folate, vitamins $\mathrm{C}$ and $\mathrm{E}$, as well as coffee, and longterm recommended treatments, including estrogen, statins, antihypertensives, and non-steroidal anti-inflammatory drugs ${ }^{6)}$ The results of the currently ongoing RCTs will probably provide a series of more scientifically based results regarding the interventions for delaying or preventing dementia.

\section{CONFLICT OF INTEREST}

No potential conflict of interest relevant to this article was reported.

\section{ACKNOWLEDGMENTS}

The author would like to acknowledge Fiona Ecarnot for her assistance with the preparation of this manuscript.

\section{REFERENCES}

1. Khachaturian ZS, Radebaugh TS. A proposed strategy for international collaborative research in brain aging and Alzheimer's disease. Int J Clin Pharmacol Res 1990;10:3-5.

2. Gauthier S, Albert M, Fox N, Goedert M, Kivipelto M, Mestre-Ferrandiz J, et al. Why has therapy development for dementia failed in the last two decades? Alzheimers Dement 2016;12:60-4.

3. World Health Organization, Alzheimer's Disease International. Dementia: a public health priority [Internet]. Geneva: World Health Organization; 2012 [cited 2016 Jul 31]. Available from: http://www.who. int/mental_health/publications/dementia_report_2012/en/.

4. Satizabal CL, Beiser AS, Chouraki V, Chene G, Dufouil C, Seshadri S. Incidence of dementia over three decades in the Framingham Heart Study. N Engl J Med 2016;374:523-32.

5. Jones DS, Greene JA. Is dementia in decline?: historical trends and future trajectories. N Engl J Med 2016;374:507-9.

6. Xu W, Tan L, Wang HF, Jiang T, Tan MS, Tan L, et al. Meta-analysis of modifiable risk factors for Alzheimer's disease. J Neurol Neurosurg Psychiatry 2015;86:1299-306.

7. Norton S, Matthews FE, Barnes DE, Yaffe K, Brayne C. Potential for 
primary prevention of Alzheimer's disease: an analysis of populationbased data. Lancet Neurol 2014;13:788-94.

8. Deckers K, van Boxtel MP, Schiepers OJ, de Vugt M, Munoz Sanchez JL, Anstey KJ, et al. Target risk factors for dementia prevention: a systematic review and Delphi consensus study on the evidence from observational studies. Int J Geriatr Psychiatry 2015;30:234-46.

9. Kivipelto M, Helkala EL, Laakso MP, Hanninen T, Hallikainen M, Alhainen K, et al. Midlife vascular risk factors and Alzheimer's disease in later life: longitudinal, population based study. BMJ 2001;322:1447-51.

10. Rusanen M, Kivipelto M, Levalahti E, Laatikainen T, Tuomilehto J, Soininen $\mathrm{H}$, et al. Heart diseases and long-term risk of dementia and Alzheimer's disease: a population-based CAIDE study. J Alzheimers Dis 2014;42:183-91.

11. Etgen T, Chonchol M, Forstl H, Sander D. Chronic kidney disease and cognitive impairment: a systematic review and meta-analysis. Am J Nephrol 2012;35:474-82.

12. Kivipelto M, Ngandu T, Laatikainen T, Winblad B, Soininen H, Tuomilehto J. Risk score for the prediction of dementia risk in 20 years among middle aged people: a longitudinal, population-based study. Lancet Neurol 2006;5:735-41.

13. Exalto LG, Quesenberry CP, Barnes D, Kivipelto M, Biessels GJ, Whitmer RA. Midlife risk score for the prediction of dementia four decades later. Alzheimers Dement 2014;10:562-70.

14. Kaffashian S, Dugravot A, Elbaz A, Shipley MJ, Sabia S, Kivimaki M, et al. Predicting cognitive decline: a dementia risk score vs. the Framingham vascular risk scores. Neurology 2013;80:1300-6.
15. Van Gool WA. Can we prevent, delay, or shorten the course of dementia? PLoS Med 2006;3:e430.

16. Andrieu S, Coley N, Lovestone S, Aisen PS, Vellas B. Prevention of sporadic Alzheimer's disease: lessons learned from clinical trials and future directions. Lancet Neurol 2015;14:926-44.

17. Kalmijn S, Launer LJ, Ott A, Witteman JC, Hofman A, Breteler MM. Dietary fat intake and the risk of incident dementia in the Rotterdam Study. Ann Neurol 1997;42:776-82.

18. Pu S, Nakagome K, Yamada T, Matsumura H, Yokoyama K, Kaneko K, et al. Association between fish consumption and prefrontal function during a cognitive task in male Japanese workers: a multi-channel near-infrared spectroscopy study. PLoS One 2015;10:e0123972.

19. Sofi F, Valecchi D, Bacci D, Abbate R, Gensini GF, Casini A, et al. Physical activity and risk of cognitive decline: a meta-analysis of prospective studies. J Intern Med 2011;269:107-17.

20. Martinez-Lapiscina EH, Clavero P, Toledo E, Estruch R, Salas-Salvado J, San Julian B, et al. Mediterranean diet improves cognition: the PREDIMED-NAVARRA randomised trial. J Neurol Neurosurg Psychiatry 2013;84:1318-25.

21. Sindi S, Mangialasche F, Kivipelto M. Advances in the prevention of Alzheimer's disease. F1000Prime Rep 2015;7:50.

22. Ngandu T, Lehtisalo J, Solomon A, Levalahti E, Ahtiluoto S, Antikainen $\mathrm{R}$, et al. A 2 year multidomain intervention of diet, exercise, cognitive training, and vascular risk monitoring versus control to prevent cognitive decline in at-risk elderly people (FINGER): a randomized controlled trial. Lancet 2015;385:2255-63. 